\title{
Techniques de dosimétrie des neutrons pour le personnel des centrales nucléaires
}

\author{
G. WOLBER (*) \\ (Manuscrit reçu le 28 juillet 1981) \\ RÉSUMÉ
}

Les neutrons détectés autour des réacteurs des centrales nucléaires proviennent des fuites à travers les ouvertures de passage de la protection biologique. Si les équivalents de dose enregistrés constituent une part très faible de l'irradiation du personnel, leur niveau nécessite leur prise en compte.

Les agents circulant ou intervenant dans les zones exposées se déplacent par rapport aux faisceaux de neutrons. Les dosimètres individuels de neutrons disponibles ne conviennent donc pas. L'estimation des équivalents de dose se fait indirectement à partir des niveaux d'irradiation mesurés avec les instruments.

On passe en revue les divers matériels utilisés ou en cours de développement et les recherches en cours sur les dosimètres individuels.

\begin{abstract}
The neutrons detected around nuclear power reactors come from leakages through the biological shield apertures. If the dose equivalents registered contribute in a very low part to workers' exposures, their level requires that they should be taken into account.

The workers walking about or operating in risk areas are moving with respect to the neutron beans. Therefore, individual neutron dosimeters are not suitable. The dose equivalents are estimated indirectly from the exposure levels measured by various instruments.

A review is made of the instruments used or being developed as well as of the present investigations carried out on individual dosimeters.
\end{abstract}

\section{INTRODUCTION}

La principale source de neutrons dans une centrale électronucléaire est le réacteur, siège des fissions induites des isotopes fissiles du combustible nucléaire. Ces neutrons sont toujours accompagnés de rayonnements gamma émis

(*) Électricité de France, Comité de Radioprotection, 39, rue de Washington, 75008 Paris. 
pendant la fission (photons gamma de fission prompts) et à la suite de la désintégration radioactive des produits de fission et d'activation à vie courte. Parmi les plus connus et les plus gênants de ces derniers figure l'azote 16, de période 7,12 secondes, qui émet des photons de 6,1 et $6,13 \mathrm{MeV}$. Ces rayonnements sont arrêtés par les protections biologiques du réacteur. Les neutrons détectés proviennent alors des fuites dans ces protections dont l'importance et la localisation sont liées à la conception de la filière. Ainsi, à la puissance nominale :

- pour les réacteurs graphite-gaz intégrés, type EDF 4 (Saint-Laurent A, Vandellos 1), les neutrons ne sont pas décelables : les débits de fluence mesurés sont inférieurs à $1 \mathrm{n} / \mathrm{cm}^{2}$.s correspondant à des débits d'équivalent de dose inférieurs à $0,1 \mu \mathrm{Sv} / \mathrm{h}(10 \mu \mathrm{rem} / \mathrm{h})$;

- pour les réacteurs à neutrons rapides à conception intégrée comme Phénix, les fuites sont faibles, très localisées et inférieures à $0,1 \mathrm{mSv} / \mathrm{h}$ $(10 \mathrm{mrem} / \mathrm{h})[1]$;

- pour les centrales $P W R$, les zones les plus exposées aux neutrons sont les casemates des pompes primaires et des générateurs de vapeur qui reçoivent directement le rayonnement du réacteur à travers les ouvertures des passages des tuyauteries dans la protection biologique avec des niveaux variant de $0,3 \mathrm{~Sv} / \mathrm{h}$ $(30 \mathrm{rem} / \mathrm{h})$ à $1 \mathrm{~Sv} / \mathrm{h}(100 \mathrm{rem} / \mathrm{h})[2]$;

- pour les réacteurs graphite-gaz prototypes de Chinon 2 et Chinon 3, les fuites sont localisées aux sorties des tuyauteries du gaz carbonique où les débits d'équivalent de dose peuvent aller jusqu'à $3 \mathrm{~Sv} / \mathrm{h}(300 \mathrm{rem} / \mathrm{h})$.

Ces niveaux sont pratiquement proportionnels à la puissance du réacteur : on n'observe les neutrons qu'en marche. Comme les faisceaux de neutrons sont très localisés, la condamnation des accès aux locaux les plus exposés, en marche, n’a pas posé de difficultés.

Les autres sources de neutrons rencontrées sur les sites électronucléaires proviennent des châteaux de transport de combustible irradié. Les neutrons ont pour origine les fissions spontanées des isotopes 240 et 242 du plutonium formé. Les niveaux sont faibles, de l'ordre de $0,1 \mathrm{mSv} / \mathrm{h}(10 \mathrm{mrem} / \mathrm{h})$.

Comme les centrales PWR constituent l'essentiel du programme français de développement des centrales nucléaires, le problème des neutrons sera exposé en détail dans le cas de cette filière. Les dispositions réglementaires adoptées ont permis de maintenir les niveaux d'irradiation par les neutrons à des valeurs très basses. La prise en compte des doses neutrons est obtenue à partir des cartographies des débits d'équivalent de dose réalisées à différents paliers de puissance au cours de la mise en service de chaque tranche des centrales. Certaines valeurs figurant sur ces cartographies ont été extrapolées à partir de mesures à faible puissance afin de limiter l'irradiation du personnel chargé d'effectuer ces mesures.

Les équivalents de dose individuels neutrons n'ont pas dépassé $5 \mathrm{mSv}$ $(0,5 \mathrm{rem})$ et les équivalents de dose collectifs représentent une part très faible (quelques pour cent) de l'irradiation totale du personnel. Les valeurs les plus élevées sont enregistrées durant les premières années de fonctionnement des 
centrales en raison de la fréquence plus élevée des interventions dans le bâtiment réacteur.

On va donc envisager successivement les méthodes employées pour le suivi dosimétrique du personnel et les recherches poursuivies dans le domaine des dosimètres individuels.

\section{MÉTHODES ACTUELLES}

\subsection{Problémes particuliers a la dosimétrie individuelle des NeUtrons en CENTRALE NUCLÉAIRE}

Il y a d'abord la nature des sources. Les faisceaux de neutrons ont une intensité proportionnelle à la puissance du réacteur avec un spectre en énergie et en direction uniquement fonction de la position du point de mesure dans le bâtiment du réacteur. L'agent, qui effectue les rondes ou accède aux lieux d'intervention, se déplace par rapport à ces sources. Il est donc soumis à des rayonnements neutroniques très variables en intensité, spectre et direction. Pour les travaux autour des châteaux de transport du combustible irradié, l'agent se déplace par rapport à des faisceaux de neutrons dont la répartition spatiale et l'intensité sont constantes.

En ce qui concerne le détecteur, on est loin de posséder le dispositif idéal. Les neutrons ne peuvent être détectés que par l'intermédiaire d'interactions nucléaires génératrices de particules chargées. Malheureusement, la réponse en énergie de ces interactions ne suit pas la loi du facteur de conversion de la fluence neutronique en équivalent de dose recommandée par la C.I.P.R. [3] ( fig. 1). Enfin, certains détecteurs sont sensibles au rayonnement gamma, introduisant une lecture parasite dans les champs mixtes neutron-gamma rencontrés sur les sites électronucléaires. On comprend pourquoi il n'existe pas encore de détecteur individuel insensible au rayonnement gamma et capable de donner, par lecture

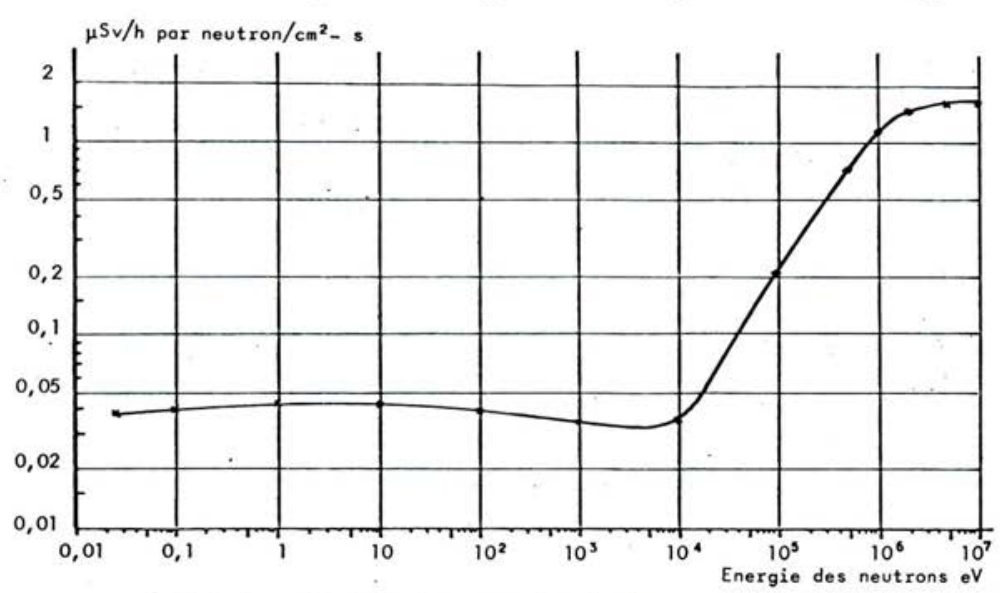

FIG. 1. - Facteur de conversion des flux neutroniques en débits d'équivalent de dose, d'après les recommandations de la C.I.P.R. [3]. 
directe et pour n'importe quel rayonnement neutronique, les équivalents de dose reçus à partir de $0,1 \mathrm{mSv}(10 \mathrm{mrem})$.

L'évaluation du risque individuel, dans toutes les conditions d'irradiation par les neutrons sur un site électronucléaire, est difficile si l'on veut la faire de façon rigoureuse. Le peu de gens irradiés et les niveaux d'équivalent de dose enregistrés permettent de se contenter de méthodes plus approchées. Nous allons les analyser du point de vue de l'appréciation de l'équivalent de dose de neutrons reçu par les personnes intervenant dans le bâtiment réacteur, en marche, dans les zones où ces équivalents de dose sont à prendre en compte.

\subsection{Le Dosimètre A ALBÉdo $[4,5]$}

Les détecteurs solides ayant une sensibilité suffisante (seuil inférieur à $0,1 \mathrm{mSv}$ ) n'ont une réponse satisfaisante qu'aux neutrons thermiques dont l'énergie est inférieure à $0,4 \mathrm{eV}$. Il faut donc thermaliser, c'est-à-dire ralentir les neutrons d'énergie plus élevée jusque dans le domaine du thermique. Ceci est obtenu de manière très simple en se servant des tissus mous du corps humain comme ralentisseur et comme rétrodiffuseur des neutrons thermalisés : c'est le principe du dosimètre à albédo, c'est-à-dire à réflexion de neutrons. Le détecteur utilisé en général est une pastille thermoluminescente au fluorure de lithium enrichi à l'isotope $6 \mathrm{du}$ lithium. La thermoluminescence est le résultat des ionisations produites par les particules alpha et les noyaux de tritium libérés par la réaction nucléaire ${ }^{6} \mathrm{Li}(n, \alpha){ }^{3} \mathrm{H}$. Ce détecteur enregistre également les doses de rayonnement gamma, avec une sensibilité de l'ordre de $1 / 20$ de celle pour les neutrons donnant des indications parasites dans les champs mixtes neutrongamma. Ce défaut est compensé en faisant une lecture différentielle avec une pastille de fluorure de lithium 7, de même sensibilité aux rayons gamma, mais 500 à 1000 fois moins sensible aux neutrons thermiques et ne donnant une indication notable que pour les neutrons d'énergie supérieure à $1 \mathrm{MeV}$. Le montage de base est formé de deux paires de pastille de fluorure de lithium 6 et 7 séparées par un écran de cadmium, ce qui fait la séparation entre les neutrons incidents et rétrodiffusés ( fig. 2). La réponse globale d'un dosimètre à albédo, en fonction de l'énergie des neutrons, ne suit pas la relation équivalent de dosefluence recommandée par la C.I.P.R. Ces dosimètres présentent une hypersensibilité aux neutrons thermiques sauf pour les modèles plus récents où l'usage d'écrans au bore leur permet d'avoir une réponse correcte entre $1 \mathrm{eV}$ et $10 \mathrm{keV}$ mais au détriment de la sensibilité globale ( $f i g .3$ ). Il s'ensuit que ces détecteurs ont une réponse très sensible au spectre et à la direction du faisceau incident ( fig. 4). Malgré ce défaut, ce dosimètre est très utilisé car il est très sensible avec un seuil de détection inférieur au millirem, facile à mettre en œuvre et il donne de bonnes indications pour les postes de travail fixes moyennant un bon étalonnage. Mais pour les raisons évoquées ci-dessus, il est inutilisable pour le personnel des sites électronucléaires.

\subsection{L'émulsion photographique Kodak NTA [4]}

L'émulsion nucléaire photographique Kodak NTA est d'usage courant en France, au C.E.A. et à la COGEMA. Son usage est exclu en centrale nucléaire 
car elle est sensible d'une part aux rayonnements gamma et, d'autre part, son seuil de détection en énergie pour les neutrons est de l'ordre de $700 \mathrm{keV}$, là où leur

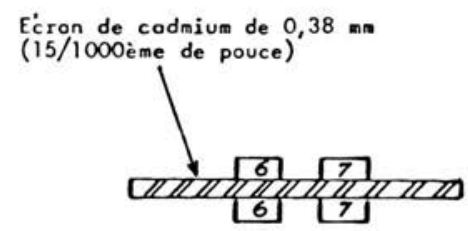

a) Montage de base (Hankins)

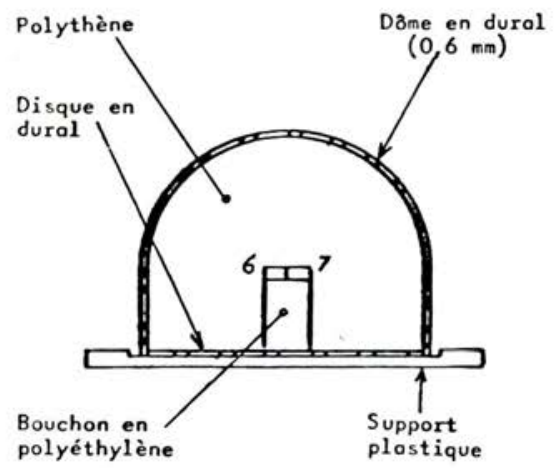

c) Dosimètre de Burger

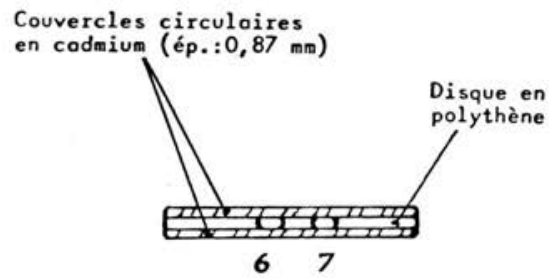

e) Dosimètre de Preston

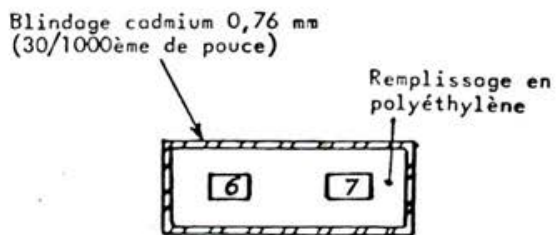

b) Dosimètre de Hankins

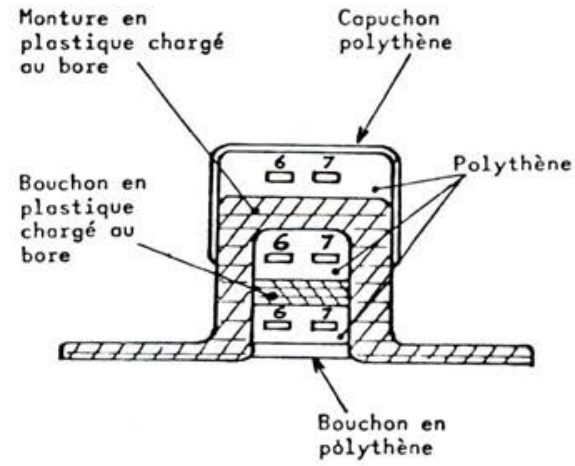

d) Dosimètre de Piesch

FIG. 2. - Quelques exemples de réalisations de dosimètres à albédo, d'après [4] et [5]. 6, pastille thermoluminescente au fluorure de lithium $6 ; 7$, pastille thermoluminescente au fluorure de lithium 7. 
contribution à l'équivalent de dose, dans les centrales, commence à décroître rapidement.

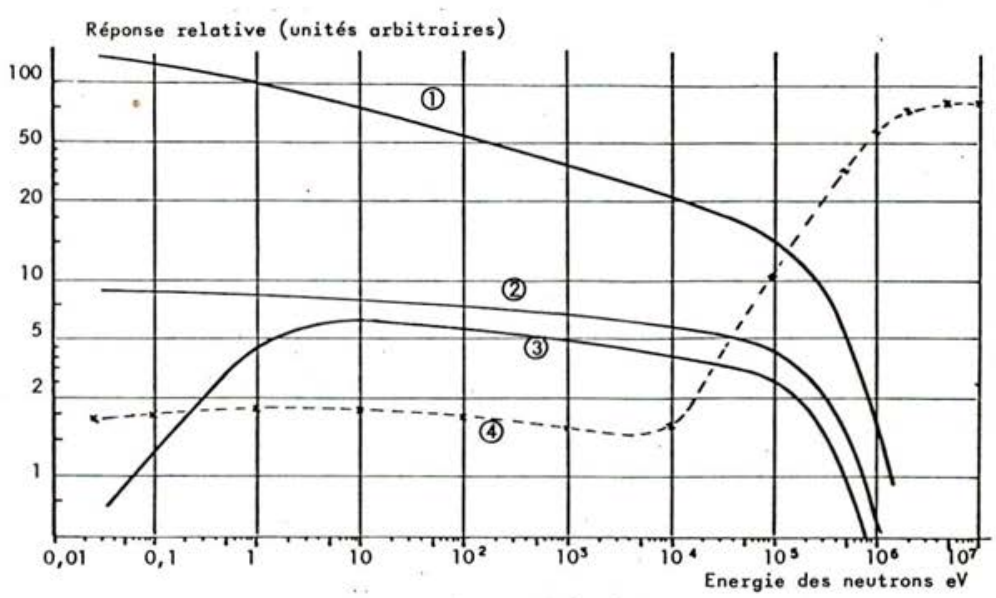

FIG. 3. - Courbes de réponse typiques de dosimètres à albédo avec leur sensibilité relative, d'après [5].

1. dosimètre de Burger (R.F.A.); 2, dosimètre de Piesch (R.F.A.); 3, dosimètre de Preston (Grande-Bretagne) (équivalent du dosimètre de Hankins); 4, courbe idéale de la C.I.P.R. [3].

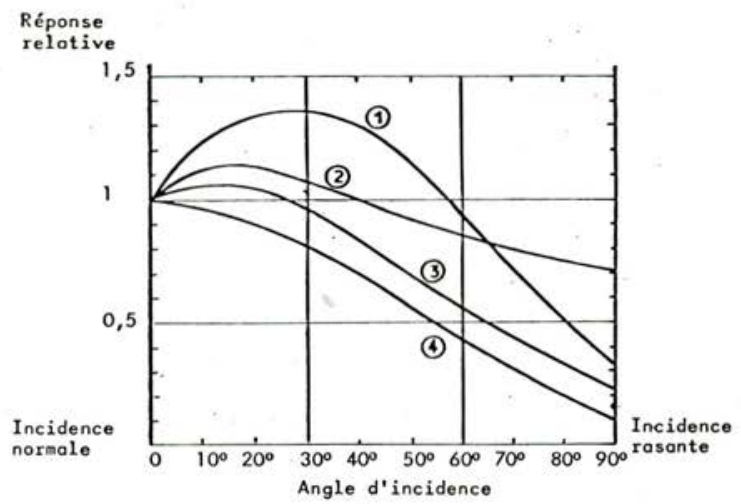

FIG. 4. - Effet de l'angle d'incidence sur la réponse aux neutrons d'un dosimètre thermoluminescent à albédo, d'après [5]. Fantôme cylindrique elliptique $20 \times 30 \mathrm{~cm}$. 1 , neutrons monoénergétiques de $1,7 \mathrm{MeV} ; 2$, neutrons de fission du ${ }^{252} \mathrm{Cf} ; 3$, neutrons rapides $(700 \mathrm{keV}) ; 4$, neutrons intermédiaires $(100 \mathrm{keV})$. 


\section{4. Évaluation des doses a PaRTIR de La dosimétrie DE ZONE}

\section{4.1. Cartographie du rayonnement neutronique}

Les niveaux d'irradiation dépendant de la puissance, les conditions de circulation dans le bâtiment réacteur en dépendent aussi. La détermination des sous-zones dans le bâtiment réacteur et l'estimation du coût dosimétrique des interventions nécessitent l'établissement d'une cartographie des débits de dose de rayonnement gamma et de neutrons. Cette opération est effectuée à puissance réduite. On mesure en chaque point les débits d'équivalent de dose de neutrons. Certains points particuliers peuvent éventuellement faire l'objet d'une spectrométrie directe afin de vérifier les protections biologiques.

\subsubsection{Les appareils (fig. 5)}

Les appareils disponibles sur le marché pour les mesures courantes de cartographie des neutrons sont basés sur la détection des neutrons thermalisés par des sphères modératrices en polyéthylène. Ces appareils sont à peu près insensibles au rayonnement gamma.

Pour la mesure des débits de fluence et principalement la détection des fuites de neutrons, on utilisait, à E.D.F., le Maneutron à compteur proportionnel au trifluorure de bore [réaction ${ }^{10} \mathrm{~B}(n, \alpha) /{ }^{7} \mathrm{Li}$ ] et sphère modératrice de $10.7 \mathrm{~cm}$ de diamètre $(4,2$ pouce). La mesure est omnidirectionnelle. La réponse en énergie est constante à $\pm 10 \%$ pour les neutrons de $100 \mathrm{eV}$ à $1 \mathrm{MeV}$ et à $\pm 30 \%$ de $0,5 \mathrm{eV}$ à $3 \mathrm{MeV}$. Le modèle le plus sensible a un seuil de détection de $10 \mathrm{n} / \mathrm{cm}^{2} . \mathrm{s}$ et une lecture linéaire jusqu'à $5 \cdot 10^{4} \mathrm{n} / \mathrm{cm}^{2}$. $\mathrm{s}$. Il sature au-delà mais garde la pleine déviation pour des débits de fluence 100 fois plus élevés. Ces valeurs sont à multiplier par 10 pour le modèle à haut flux. Le facteur de conversion du débit de fluence neutronique en débit d'équivalent de dose varie de 50 à $250 \mathrm{n} / \mathrm{cm}^{2}$. s par $\mathrm{mrem} / \mathrm{h}$ suivant les points de mesure. Les facteurs de conversion les plus bas indiquent un faisceau riche en neutrons rapides donc provenant d'une fuite proche du point de mesure alors que les valeurs les plus élevées correspondent à des spectres de neutrons en majorité diffusés comme en zone annulaire. Le fluxmètre Maneutron est donc impropre à donner des indications sur les débits d'équivalent de dose en tout point du bâtiment réacteur et son usage est déconseillé en dosimétrie à E.D.F.

Pour la mesure des débits d'équivalent de dose, l'appareil le plus répandu à E.D.F. est le rem-compteur Andersson-Braun modifié par Hankins [6] et fabriqué par Studsvik (modèle 2002 D). Le détecteur à trifluorure de bore est placé dans un cylindre de polyéthylène limité par un écran cylindrique perforé en plastique chargé au bore. Le tout est placé dans l'axe d'un cylindre modérateur en polyéthylène de $215 \mathrm{~mm}$ de diamètre sur $230 \mathrm{~mm}$ de long tronqué à l'avant par une surface sphérique. La réponse en énergie d'un tel système suit la relation équivalent de dose-fluence de la C.I.P.R. à mieux qu'un facteur 2 ( $f$ ig. 6) pour des neutrons d'énergie de $0,01 \mathrm{eV}$ à $10 \mathrm{MeV}$. Contrairement aux appareils à sphère, il y a une baisse de sensibilité propre de $50 \%$ vers l'arrière de l'appareil, à l'opposé de la surface sphérique. L'indication est donnée sur un cadran à échelle logarithmique à cinq décades couvrant la gamme de $0,1 \mathrm{mrem} / \mathrm{h}\left(10^{-6} \mathrm{~Sv} / \mathrm{h}\right)$ à $10 \mathrm{rem} / \mathrm{h}(0,1 \mathrm{~Sv} / \mathrm{h})$. Le rem-compteur Andersson-Braun a pour inconvénient 
d'être lourd $(11 \mathrm{~kg})$ et encombrant mais il donne les mesures de débit d'équivalent de dose à $10 \%$ près en toute zone. Il n'est plus commandé de matériel neuf de ce type. Il sera remplacé par le Cramal au fur et à mesure des renouvellements.

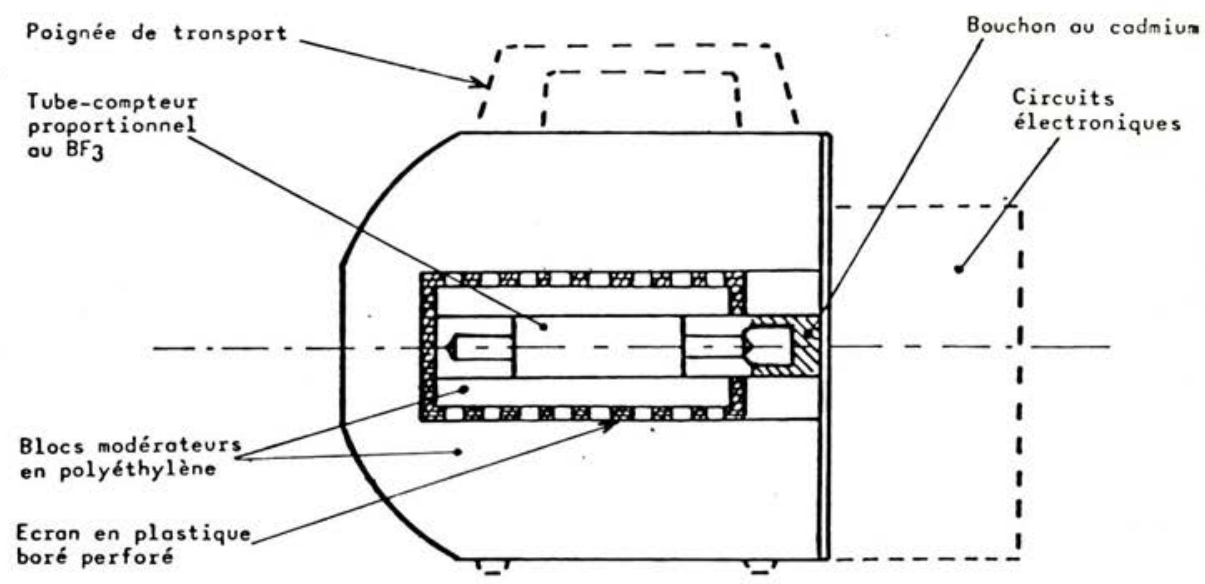

a) Compteur Andersson-Braun modifié.

Réalisation Studsvik. Masse : $11 \mathrm{~kg}$.

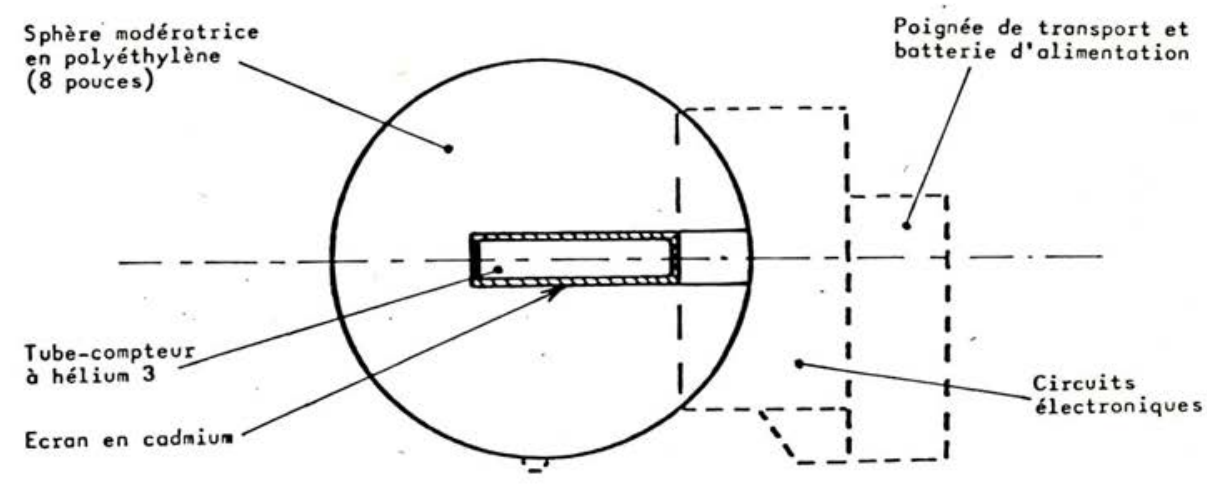

b) CRAMAL (CEA). Masse : $6 \mathrm{~kg}$.

FIG. 5. - Rem-compteurs à modérateur, schémas de principe.

Représentation à la même échelle. 


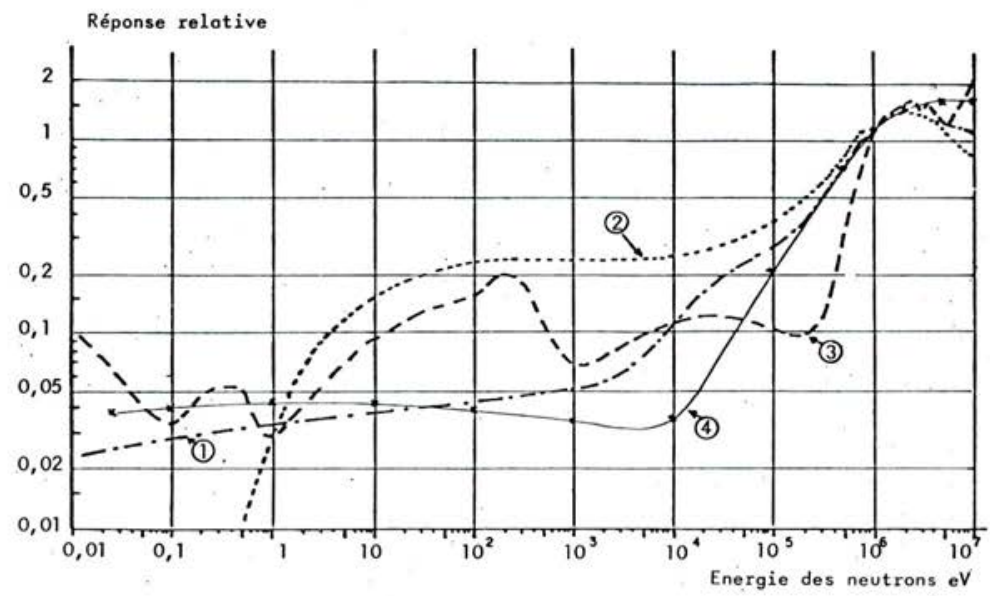

FIG. 6. - Réponse en énergie des divers rem-compteurs. 1, Andersson-Braun, modèle Studsvik 2002-D (notice constructeur); 2, Cramal 1, d'après [7], extrapolation en dessous de $30 \mathrm{keV}$, d'après [15]; 3, RIF, d'après [14]; 4, loi de variation du facteur de conversion flux-débit d'équivalent de dose de la C.I.P.R., d'après [3].

Le compteur de rems à modérateur allégé ou Cramal [7], développé par le C.E.A. au Centre d'études nucléaires de Grenoble, mesure les débits d'équivalent de dose et les équivalents de dose cumulés pendant la mesure, ainsi que le temps de mesure. Il comporte un compteur proportionnel à hélium 3 sous écran de cadmium, utilisant la réaction ${ }^{3} \mathrm{He}(n, p){ }^{3} \mathrm{H}$, et placé au centre d'une sphère modératrice de $20 \mathrm{~cm}$ de diamètre ( 8 pouces). La réponse en énergie de cet ensemble est proche de la relation équivalent de dose-fluence de la C.I.P.R. d'un facteur variant de 1 à 2 pour des neutrons de $2 \mathrm{keV}$ à $15 \mathrm{MeV}$. Si l'usage du cadmium a permis d'alléger la sphère modératrice, il semble qu'on ne puisse pas aller très loin dans cette voie. L'affichage est sous forme numérique par cristaux liquides. Il permet les mesures d'équivalents de dose cumulés jusqu'à $100 \mathrm{rem}$ (1 Sv), des débits d'équivalent de dose de $0,1 \mathrm{mrem} / \mathrm{h}\left(10^{-6} \mathrm{~Sv} / \mathrm{h}\right)$ à $100 \mathrm{rem} / \mathrm{h}$ $(1 \mathrm{~Sv} / \mathrm{h})$ pour des temps cumulés jusqu'à 10 heures. Il est plus léger $(6,3 \mathrm{~kg})$ et plus pratique, par son type d'affichage, que le compteur Andersson-Braun pour les mêmes services rendus. Le Cramal est fabriqué industriellement et fait partie des appareils recommandés pour la mesure des neutrons à E.D.F.

\subsubsection{Prise en compte des doses}

Le bilan dosimétrique est estimé avant toute intervention en zone contrôlée. Pour les neutrons, cette estimation est obtenue à partir de la cartographie des débits d'équivalent de dose corrigés de la puissance du réacteur et d'après les temps de séjour et d'accès aux divers lieux de travail. Au cours de l'intervention, la dosimétrie est évaluée d'après les temps réels et d'après les indications du ou 
des rem-compteurs emportés par l'équipe. On procède d'une façon analogue autour des châteaux de transport du combustible irradié.

\section{RECHERCHES EFFECTUÉES SUR LES DOSIMĖTRES INDIVIDUELS}

\subsection{But et NATURE DES RECHERCHES EFFECTUÉES}

On cherche à obtenir des dosimètres capables, sinon par simple lecture, du moins par un traitement numérique approprié, de donner l'équivalent de dose pour tout le spectre d'énergie des neutrons rencontrés sur les sites nucléaires, c'est-à-dire des neutrons thermiques aux neutrons rapides de 2 à $5 \mathrm{MeV}$. Cette caractéristique est la condition pour que la réponse du détecteur ne dépende pas de la direction et du spectre de la source. Afin d'éviter un traitement complexe, ces détecteurs doivent être peu ou pas sensibles au rayonnement gamma qui accompagne le rayonnement neutronique. Enfin, le système de lecture doit être capable de traiter entre quelques centaines et quelques milliers de détecteurs par mois, donc être automatisé.

Les deux interactions nucléaires utilisées sont les collisions élastiques neutron-proton [réaction $(n, p)$ collision] pour les neutrons d'énergie supérieure à $100 \mathrm{keV}$ et les réactions $(n, \alpha)$ pour les neutrons d'énergie inférieure à $50 \mathrm{keV}$. On a donc deux classes de détecteurs : les dispositifs à protons de recul des réactions $(n, p)$ et les dispositifs à convertisseur $(n, \alpha)$. L'état d'avancement de toutes ces recherches est au stade des études de faisabilité pour un prototype industriel.

\subsection{DÉtecteurs a PROTONS DE RECUl}

\subsubsection{Caractéristiques générales}

Le corps humain constitue un diffuseur important pour les neutrons incidents en renvoyant un spectre dégradé par rapport au spectre du faisceau incident, donc plus pauvre en neutrons intermédiaires et rapides. Les détecteurs à protons de recul ont donc une réponse beaucoup moins sensible à la direction de la source que les détecteurs à neutrons thermiques. L'étalonnage se fait avec le dosimètre placé sur un fantôme et face à la source mais, il faudra envisager des mesures en incidence rasante.

\subsubsection{Détecteurs à émission exoélectronique thermostimulée (EETS)}

Cette technique est en cours d'étude au Service technique d'équipements de protection et de dosimétrie (S.T.E.P.D.) à l'Institut de protection et de sûreté nucléaire (I.P.S.N.) du C.E.A. à Fontenay-aux-Roses en liaison avec le Physikalisch-Technische Bundesanstalt Institut (P.T.B.) de Berlin. L'émission exoélectronique thermostimulée a la même origine que la thermoluminescence : les électrons arrachés par les particules chargées incidentes et piégés dans les couches superficielles du détecteur (épaisseur : quelques nanomètres) sont libérés par chauffage, accélérés dans un champ électrique et comptés [8]. Dans le cas des neutrons, les particules chargées sont les protons de recul provenant d'un radiateur hydrogéné plaqué contre la face émissive. Le matériau émissif est 
de l'alumine $\mathrm{Al}_{2} \mathrm{O}_{3}$ ou de l'oxyde de béryllium $\mathrm{BeO}$ frittés et déposés sur un substrat en acier inoxydable ou en graphite. Des recherches s'effectuent aussi sur le fluorure de lithium 7 (Fli) déjà connu pour ses propriétés thermoluminescentes [9]. La faible épaisseur de la couche émissive permet la détection de protons de recul de faible énergie, de 5 à $10 \mathrm{keV}$, ce qui laisse prévoir la détection de neutrons d'au moins $100 \mathrm{keV}$ et jusqu'à $2 \mathrm{MeV}$. En réalisant un sandwich de radiateurs et de matériaux émetteurs, il semble possible de réaliser un dosimètre donnant des équivalents de dose. Le seuil de détection théorique est de $0,1 \mathrm{mSv}(10 \mathrm{mrem})$ pour l'alumine, plus bas pour l'oxyde de béryllium, et la réponse en équivalent de dose peut être linéaire jusqu'à $0,5 \mathrm{~Sv}$ ( $50 \mathrm{rem})$. Pour le fluorure de lithium, les recherches sont moins avancées en EETS. Ces détecteurs sont évidemment sensibles aux rayonnements gamma, de l'ordre de 5 à 10 fois plus, mais on peut diminuer ce rapport en plaçant le dosimètre dans un boîtier en plomb.

\subsection{3. Émulsions nucléaires à lecture par activation}

Cette technique est étudiée au Service d'application des détecteurs visuels (SADVI) de l'Université Louis-Pasteur à Strasbourg. Le détecteur est une plaque photographique placée contre un radiateur hydrogéné. Les traces de protons de recul dans l'émulsion photographique sont activées, après développement, par une source de neutrons. On mesure ensuite l'activité de l'isotope $110 \mathrm{~m}$ de l'argent par spectrométrie gamma. Cette méthode permet d'améliorer le rapport signal-bruit par rapport à la lecture optique. Le seuil de détection est de $0,1 \mathrm{mSv}(10 \mathrm{mrem})$ de neutrons épithermiques dans un rayonnement gamma ambiant donnant une dose cumulée de 3 à $4 \mathrm{mSv}$ (300 à $400 \mathrm{mrem}$ ). La réponse en énergie est actuellement constante de $100 \mathrm{keV}$ à $2 \mathrm{MeV}$ mais peut être rendue conforme à celle du facteur de conversion de la C.I.P.R. La dynamique en dose cumulée est celle des films, ce qui obligera à disposer de deux émulsions pour couvrir la gamme $10 \mathrm{mrem}-1 \mathrm{rem}$.

\subsubsection{Détecteurs à traces de protons de recul}

Les études se font aussi au S.T.E.P.D. à Fontenay-aux-Roses. Le principe est basé sur la révélation des traces de protons de recul faites dans divers matériaux plastiques tels le nitrate de cellulose, CN 85 (études en liaison avec l'Université de Limoges) et le diallyl-glycol, CR 39 (études en liaison avec l'Académie des Sciences de Tchécoslovaquie). Ces protons proviennent d'un radiateur hydrogéné au polyéthylène placé contre le matériau détecteur qui peut aussi engendrer lui-même des protons de recul. La révélation est obtenue par attaque électrochimique différentielle à la soude ou à la potasse caustique avec un champ électrique alternatif à haute fréquence $(2 \mathrm{kHz})$. Les traces, plus vite attaquées que le restant du matériau, apparaissent sous forme de trous qu'on peut dénombrer au microscope optique ou au quantimètre (écran de télévision avec traitement d'image). On peut faire aussi une lecture automatique par étincelage selon la méthode de Tommasino [4]. Le détecteur est pris en sandwich entre deux électrodes en mylar métallisé mises progressivement sous tension. Des claquages se produisent au niveau des traces qui volatilisent le métal de sorte qu'il y a une étincelle par trace $[10,11]$. En comptant les claquages on compte les 
trous et ceci pour toute la surface du détecteur. Le seuil de détection attendu est de l'ordre de 0,2 à $0,5 \mathrm{mSv}$ (20 à $50 \mathrm{mrem}$ ) avec un radiateur hydrogéné et la réponse est linéaire jusqu'à environ $1 \mathrm{~Sv}$ (100 rem). Pour des doses plus élevées, il y a saturation par recouvrement des traces. La réponse en énergie d'un détecteur est plate de $100 \mathrm{keV}$ à $2 \mathrm{MeV}$ pour un détecteur nu de $5 \mu \mathrm{m}$ d'épaisseur, mais on peut avoir les équivalents de dose en composant des sandwichs de radiateurs et de détecteurs. Les détecteurs à traces sont insensibles au rayonnement gamma dans la gamme des doses qui concernent la radioprotection. Par contre, il y a une perte de sensibilité de $90 \%$ quand l'angle d'incidence des faisceaux de neutrons passe de 0 (incidence normale) à $90^{\circ}$ (incidence rasante) [12].

\subsection{Détecteurs a CONVERTISSEURS A RÉACTION NUCLÉAire}

\subsubsection{Généralités}

Les convertisseurs sont utilisés dans le domaine des énergies des neutrons thermiques et épithermiques (énergie $<1 \mathrm{keV}$ ) où les protons de recul ne peuvent plus être détectés. Ces convertisseurs font appel aux réactions de capture avec émission de photons $\gamma$ (capture radiative) ou de particules alpha (réaction $n, \alpha$ ), générant des particules détectables pour ces catégories de neutrons. On ne parlera pas des détecteurs à convertisseur ( $n$, Fission) dont le plus intéressant est celui au ${ }^{237} \mathrm{~Np}[4,13]$. Sa réponse en énergie, pour les neutrons, suit assez bien la relation équivalent de dose-fluence recommandée par la C.I.P.R. et il est peu sensible au rayonnement gamma. En France, il n'est pas question de le faire porter, son principal inconvénient étant d'irradier de façon non négligeable son porteur.

\subsubsection{Dosimètre-fılm à écran de cadmium}

C'est le premier dosimètre de neutrons mis en œuvre par le C.E.A. Ce dispositif a été utilisé dans les premiers dosimètres à albédo. Les photons gamma de capture radiative,émis par le cadmium, situés dans un spectre allant de 0,5 à $3 \mathrm{MeV}$ impressionnent une émulsion photographique placée contre l'écran. Ce détecteur n'est sensible qu'aux neutrons thermiques d'énergie inférieure à $0,4 \mathrm{eV}$, sa limite de détection est de $0,02 \mathrm{mSv}(2 \mathrm{mrem})$ dans un rayonnement gamma de $1 \mathrm{mSv}(100 \mathrm{mrem})$ mais l'imprécision est grande quand la dose de rayonnement gamma approche les $30 \mathrm{mSv}$ ( $3 \mathrm{rem}$ ). Le SADVI envisage de l'utiliser en complément du film à protons de recul pour réaliser la correction de la composante des neutrons thermiques dans le spectre global. Pour éviter l'effet d'albédo du porteur, on peut interposer un écran à base de bore ou de lithium.

\subsubsection{Dosimètres thermoluminescents sous blindage de cadmium (fig. $2 b$ )}

Ce détecteur est en cours de développement au C.E.A., S.T.E.P.D., Cadarache. Il était prévu à l'origine pour les personnes travaillant sur les éléments transuraniens, mais les études se poursuivent aussi pour son utilisation en centrale nucléaire. Il s'apparente au dosimètre individuel de HaNKINS [4]. C'est un détecteur thermoluminescent au fluorure de lithium 6 dont le principe de conversion par la réaction $(n, \alpha)$ est le même que pour le dosimètre à albédo. Le dosimètre est constitué de quatre pastilles thermoluminescentes au fluorure de lithium, une enrichie en lithium 6 , deux en lithium 7 et une au lithium naturel 
montées dans un boîtier standard, modèle PGP 1, le tout enfermé dans un boîtier en cadmium de $0,7 \mathrm{~mm}$ d'épaisseur et d'un boîtier en plomb d'environ $1 \mathrm{~mm}$ d'épaisseur destiné à arrêter les photons émis par les transuraniens. La lecture se fait comme pour le dosimètre à albédo mais, du fait qu'il ne détecte pas les neutrons thermiques, l'effet d'albédo est bien moins marqué. De ce fait, sa réponse en énergie est constante à $30 \%$ près pour des neutrons entre $1 \mathrm{eV}$ et $50 \mathrm{keV}$ et il est peu sensible à la direction du faisceau incident. Dans un spectre de neutrons du type rayonné par les réacteurs PWR, la lecture est pratiquement proportionnelle à l'équivalent de dose mais le seuil de détection est ramené à la plage de 0,3 à $0,5 \mathrm{mSv}$ ( 30 à $50 \mathrm{mrem}$ ).

\subsubsection{Dosimètres à traces à convertisseur $(n, \alpha)$}

Les études sont suivies en France au C.E.A., S.T.E.P.D., à Fontenay-auxRoses. Le matériau est le même que pour les détecteurs à protons de recul : CN 85, CR 39 ou LR 115 de Kodak, autre nitrate de cellulose couché sur support inerte. Le convertisseur est placé contre le détecteur sur une face. Il est, soit en tétraborate de lithium $\mathrm{Li}_{2} \mathrm{~B}_{4} \mathrm{O}_{7}$, lithium et bore naturels, soit au fluorure de lithium 6, soit en bore naturel avec une sensibilité alors quadruplée. La lecture se fait de la même manière que pour les dosimètres à traces de protons de recul. La loi de réponse en énergie est du type $1 / v$ c'est-à-dire qu'il y a hypersensibilité pour les neutrons thermiques et le comportement de ce détecteur se rapproche du dosimètre à albédo. Pour compenser cette hypersensibilité, le dosimètre est enveloppé de plaques de plexiglas avec des zones couvertes de cadmium. On a ainsi deux zones répondant, l'une aux neutrons thermiques, l'autre aux neutrons épithermiques. Associé au détecteur à traces de protons de recul, il peut constituer un dosimètre donnant les équivalents de dose pour tous les spectres d'énergie et absolument insensible au rayonnement gamma.

\subsection{Perspectives}

Ces recherches sont, suivant les cas, plus ou moins proches d'aboutir à un prototype. Elles doivent établir lesquels de ces systèmes sont susceptibles de devenir opérationnels. Au cas où ce serait possible, un développement industriel peut être envisagé d'ici quelques années.

\section{RECHERCHES POURSUIVIES DANS LE DÉVELOPPEMENT DES REM-MÈTRES}

En attendant la réalisation d'un dosimètre individuel pour les neutrons utilisable en centrale nucléaire, le C.E.A. cherche à développer des rem-mètres aussi performants que le modèle d'Andersson-Braun ou le Cramal mais plus légers, car ils doivent être portatifs, à lecture directe des équivalents de dose cumulés et susceptibles d'une fabrication industrielle autorisant une maintenance suffisante pour assurer les besoins des sites en exploitation. Le S.T.E.P.D. à Cadarache a ainsi défini et réalisé le rem-compteur intégrateur à fission (RIF).

VOL. $16-\mathrm{N}^{0} 4$ 
Le RIF utilise une chambre à fission multiélectrodes à dépôt de $0,7 \mathrm{~g}$ d'uranium 235 et blindée par une enveloppe en cadmium perforée. La conception de cette chambre dérive des détecteurs de l'hodoscope du réacteur Cabri dont le rôle est de localiser les déformations des éléments combustibles essayés [14]. On obtient une courbe de réponse en énergie des neutrons qui suit la loi recommandée par la C.I.P.R. avec un facteur qui varie au maximum entre 0,5 et 2 pour des énergies de neutrons entre $0,01 \mathrm{eV}$ et $10 \mathrm{MeV}$ et qui donne les équivalents de dose à $10 \%$ près pour les spectres rencontrés sur les sites électronucléaires. La gamme de débits d'équivalent de dose couverte est de $0,01 \mathrm{mSv} / \mathrm{h}(1 \mathrm{mrem} / \mathrm{h})$ à $1 \mathrm{~Sv} / \mathrm{h}(100 \mathrm{rem} / \mathrm{h})$ avec une réponse proportionnelle à l'équivalent de dose. Le détecteur est insensible au rayonnement gamma d'énergie inférieure à $5 \mathrm{MeV}$, seuil des photofissions, mais on n'a pas rencontré ce problème au cours des essais en centrale nucléaire. Très maniable, avec une masse de $2 \mathrm{~kg}$ et une longueur de $35 \mathrm{~cm}$, cet appareil ne sera toutefois pas d'une large diffusion à cause de son prix élevé (1,5 fois celui du Cramal) et de l'impossibilité de construire les chambres à fission hors du C.E.A., parce qu'il nécessite l'usage d'une quantité importante de matière fissile. Son usage, en France, sera sans doute réservé à des mesures ponctuelles de laboratoire ou d'installations.

Le RIF dispose des mêmes indications que le Cramal dont il utilise les échelles de comptage. Il donne par simple commutation, le débit d'équivalent de dose, l'équivalent de dose cumulé et le temps de mesure. Il peut être équipé d'alarmes à seuil.

\section{CONCLUSIONS}

Il n'existe pas, à l'heure actuelle, de dosimètre individuel pour les neutrons utilisable en centrale nucléaire où les agents exposés à ces particules n'occupent pas des postes de travail fixes. Ceci explique l'effort accompli dans le développement des rem-mètres, les seuls sur lesquels on puisse compter, et qui doit aboutir, à court terme, à un matériel maniable, fiable et pratique. Malgré son imprécision, la dosimétrie effectuée à partir des indications des rem-mètres semble suffisante car les équivalents de dose individuels et collectifs pour les neutrons sont bien inférieures à ceux enregistrés pour les rayonnements gamma. Il faut remarquer qu'elle constitue une servitude d'emploi dont les dosimètres individuels permettront de s'affranchir, ce qui justifie l'intérêt des efforts de recherche poursuivis dans le domaine des détecteurs individuels dont on espère l'aboutissement d'ici la fin de cette décennie.

\section{REMERCIEMENTS}

Je tiens à remercier MM. Bermann, Medioni, Mourgues, Petel, Portal et Prigent du C.E.A., S.T.E.P.D., M. Le Tanh du C.E.A., C.E.N. Saclay, pour avoir bien voulu me communiquer les renseignements dont j'ai eu besoin, M. De Choudens du C.E.N. de Grenoble et M. Pericart de la Direction des Études et Recherches d'E.D.F. pour avoir bien voulu me conseiller sur la rédaction de cet article. 


\section{BIBLIOGRAPHIE}

[1] Quatre années de radioprotection, Rapport commun C.E.A.-E.D.F., centrale Phénix, 1974-1978.

[2] Wolber G. Le risque neutron en centrale mucléaire PWR. Dosimétrie et moyens de protection, Rapport présenté à la réunion du Comité de Radioprotection du 28 février 1980. Note interne E.D.F. D 87-15918.

[3] International Commission on Radiological Protection (I.C.R.P.), Data for protection against ionizing radiation from external sources, Supplement to I.C.R.P. publication 15, I.C.R.P. publication 21, Oxford, Pergamon press, 1973.

[4] Griffith R. V., Hankins D. E., Gammage R. B., Tommasino L., Wheeler R. V. Recent developments in personnel neutron dosimeters, a review. Health Phys., 1979, 36, 235-260.

[5] Douglas J. A., Marshall. M. The responses of some TL Albedo neutron dosimeters. Health Phys., 1978, 35, 315-324.

[6] HANKinS D. E. A modified A-B-remmeter with improved directional dependance and thermal neutron sensitivity. Health Phys., 1978, 34, 249-254.

[7] Herbaut Y., BORY P., VIALA R. Essais physiques du prototype du compteur de rem à modérateur allégé, ıype Cramal. Rapport C.E.A. (à paraitre).

[8] Petel M., Holzapfel G. Dosimétrie par émissions exoélectroniques stimulées. In $8^{\complement}$ Congrès international de la Société Française de Radioprotection, Saclay, 23-26 mars 1976, 342-363.

[9] TATAH B., Herbaut Y. Dosimétrie par émission exoélectronique thermo-stimulée au fluorure de lithium. Rapport davancement au $1^{\text {er }}$ juin 1980, Communication personnelle.

[10] LE TANH P., NIPKAY P. Un dispositif de comptage à décharges disruptives permettant d’atténuer les décharges disruptives multiples. In Solid state nuclear track detectors, Lyon, 2-6 July 1979 (François H. et al., éd.) Oxford. Pergamon press, 1980, 337-362.

[11] Decossas J. L., Vareille J. C., Moliton J. P., Teyssier J. L., Delaunay B. Enregistrements et traces de protons de type cellulosique. Nucl. Instr. Methods, 1980, 171, 319.

[12] Benton E. V., Oswald R. A., Frank A. L., Whecler R. V. Proton recoil neutron dosimeter for personnel monitoring. Health Phys., 1981, 40, 801-809.

[13] Cross W. G., ING H. The use of ${ }^{237} \mathrm{~Np}$ in personal dosimeters for fast neutrons. Health Phys., $1975,28,511-520$.

[14] Mourgues M. Utilisation de chambres à fission de grande sensibilité pour la spectrométrie et la dosimétrie des neutrons (IAEA SM 229/84). In Symposium on advances in radiation protection monitoring. Stockholm, 26-30 juin 1978, Vienne: A.I.E.A., 1979, 183-195.

[15] LYMBERIS C. Étude du flux thermique et épithermique produit dans les sphères de polyéthylène par des neutrons incidents de $10 \mathrm{eV}$ à $14 \mathrm{MeV}$ : application à la dosimétrie. Rapport C.E.A.R-4597, 1974. 